\title{
Numerical Analysis of Extensible Shearable Elastica by Direct Energy Minimization*
}

\author{
Toshimi TAKI ${ }^{\dagger}$ \\ Aerospace Company, Kawasaki Heavy Industries, Ltd., Kakamigahara, Gifu 504-8710, Japan
}

\begin{abstract}
The author and K. Kondo derived an expression for the potential energy of extensible shearable elastica based on Engesser's approach in a previous paper. In this paper, a simple method to calculate the post-buckling deformation of extensible shearable elastica is proposed. The potential energy is directly minimized by the "Solver" optimization tool of MS-Excel. The compression buckling loads calculated by the method are consistent with the buckling load equation in the previous paper. The relationship between applied load and deflection is calculated for beams with representative combinations of axial stiffness and shear stiffness. Snap-through behavior is observed in the load-displacement curves of beams with low axial stiffness.
\end{abstract}

Key Words: Extensible Shearable Elastica, Engesser's Approach, Variational Principle, Buckling Load, Post-buckling Behavior

\section{Nomenclature}

A: cross-sectional area of beam before deformation

E: Young's modulus

$G$ : shear modulus

$\boldsymbol{g}_{x}, \boldsymbol{g}_{z}$ : base vectors in $x-z$ coordinate system

$\boldsymbol{G}_{x 0}, \boldsymbol{G}_{z 0}$ : covariant base vectors on the beam axis after deformation

$\boldsymbol{G}^{x 0}, \boldsymbol{G}^{z 0}$ : contravariant base vectors after deformation

$\boldsymbol{G}_{x}, \boldsymbol{G}_{z}$ : covariant base vectors after deformation

I: moment of inertia of cross-section

$k$ : shear correction factor

$l_{0}$ : beam length before deformation

$L_{e}$ : length of beam element before deformation

$\breve{M}_{y}$ : bending moment acting on deformed crosssection (Engesser's approach)

$\breve{N}_{x}$ : axial force acting on deformed cross-section (Engesser's approach)

$P_{E n(n)}$ : buckling load of $n$-th mode of extensible shearable beam, Engesser's approach

$P_{E}$ : Euler buckling load of first mode

$\breve{Q}_{z}$ : shear force acting on deformed cross-section (Engesser's approach)

$\boldsymbol{r}_{0}(x)$ : position vector of beam axis before deformation

$\boldsymbol{r}(x, z)$ : position vector before deformation

$\boldsymbol{R}_{0}(x)$ : position vector of beam axis after deformation

$\boldsymbol{R}(x, z)$ : position vector after deformation

$\boldsymbol{u}_{0}(x)$ : displacement vector of beam axis

$\boldsymbol{u}(x, z)$ : displacement vector

(C) 2017 The Japan Society for Aeronautical and Space Sciences

*Presented at the 52nd JSASS/JSME/JAXA Structures Conference, July 21-23, 2010, Tottori, Japan.

Received 19 May 2016; final revision received 2 February 2017; accepted for publication 17 March 2017

†Corresponding author, taki_toshimi@khi.co.jp
$U$ : strain energy

$u, w$ : displacements in global coordinate system

$u_{e}, w_{e}$ : displacements in element coordinate system

$x, y, z$ : global coordinate system

$x_{e}, z_{e}$ : element coordinate system before deformation

$\breve{\varepsilon}_{x 0}, \breve{\gamma}_{x z 0}, \breve{\kappa}_{y}$ : generalized strain (Engesser's approach)

$\gamma$ : shear deformation angle of deformed crosssection

$\phi_{e}$ : inclination angle of deformed element

$\theta_{0}$ : rotation of deformed beam axis

$\Lambda_{e}$ : distance between grid points of beam element after deformation

$\Pi$ : potential energy Subscripts

$\xi_{e}, \varsigma_{e}$ : element coordinate system after deformation

0 : beam axis

1,2: grid point number

$e$ : element

E: Euler

En: Engesser

\section{Introduction}

Extensible shearable elastica is a model of a beam with axial, bending and shear deformation. Taki and Kondo ${ }^{1)}$ derived the variational principles of extensible shearable elastica expressed in the extensional strain and rotation of the beam axis, and the shear angle of the cross-section. In the derivation of the principle of stationary potential energy, Engesser's approach was used to express the components of the resultant forces working on the cross-section. An exact expression of the buckling load was obtained in the paper.

Magnusson et al. ${ }^{2)}$ analytically solved the deformation of extensible elastica and found that the buckling load of a beam with low axial stiffness becomes unstable. A snapthrough behavior occurs. Kuramoto and Kondo ${ }^{3)}$ also solved extensible elastica using the finite element method, but they 
did not notice existence of the unstable condition. Humer ${ }^{4)}$ analytically solved the extensible shearable elastica of Haringx's approach.

In order to analyze the deformation of extensible shearable elastica of Engesser's approach, a new and simple method based on the principle of energy is introduced in this study. This is a variation of the methods developed for structural analysis by the author. ${ }^{5)}$ The method is outlined as follows.

(1) A beam is divided into finite elements and each element has two grid points at both ends.

(2) The strain energy of the deformed element is expressed using the displacements of the grid points.

(3) The potential energy of the deformed beam is expressed using the displacements of the grid points of all the elements and the external force.

(4) The potential energy is calculated for assumed displacements of the grid points using MS-Excel. "Solver," in MS-Excel, is used to minimize the potential energy and the solution is obtained as a set of grid point displacements.

The method is successfully applied to the analysis of extensible shearable elastica.

\section{Formulation}

Extensible shearable elastica, shown in Fig. 1, is analyzed in this paper. The deformed geometry of a beam is shown in Figs. 2 and 3.

\subsection{Deformation of extensible shearable elastica in a global coordinate system}

According to Taki and Kondo, ${ }^{1)}$ the strain of the elastica is expressed by the displacement and rotation of the beam axis, and the shear angle of the cross-section.

The base vector of the axis after deformation is expressed as follows. The expression of $d(\quad) / d x=(\quad)^{\prime}$ is used.

$$
\begin{aligned}
\boldsymbol{u}_{0}(x) & =u \boldsymbol{g}_{x}+w \boldsymbol{g}_{z}=\boldsymbol{R}_{0}(x)-x \boldsymbol{g}_{x} \\
\boldsymbol{G}_{x 0}= & \frac{d \boldsymbol{R}_{0}}{d x} \\
= & \boldsymbol{R}_{0}{ }^{\prime} \\
= & \left(1+u^{\prime}\right) \boldsymbol{g}_{x}+w^{\prime} \boldsymbol{g}_{z} \\
= & \sqrt{G_{x x 0}}\left(\cos \theta_{0} \boldsymbol{g}_{x}+\sin \theta_{0} \boldsymbol{g}_{z}\right)
\end{aligned}
$$

where $G_{x x 0}=\boldsymbol{G}_{x 0} \cdot \boldsymbol{G}_{x 0}, u$ and $w$ are displacements of the beam axis, $\theta_{0}(x)$ is rotation of the beam axis, and $\boldsymbol{g}_{x}$ and $\boldsymbol{g}_{z}$ are base vectors of the global coordinate system. The following equations are derived from Eq. (1).

$$
\begin{aligned}
& \sqrt{G_{x x 0}}=\sqrt{\left(1+u^{\prime}\right)^{2}+\left(w^{\prime}\right)^{2}} \\
& \cos \theta_{0}=\frac{1+u^{\prime}}{\sqrt{\left(1+u^{\prime}\right)^{2}+\left(w^{\prime}\right)^{2}}}, \quad \sin \theta_{0}=\frac{w^{\prime}}{\sqrt{\left(1+u^{\prime}\right)^{2}+\left(w^{\prime}\right)^{2}}} \\
& \theta_{0}{ }^{\prime}=\frac{\left(1+u^{\prime}\right) w^{\prime \prime}-u^{\prime \prime} w^{\prime}}{\left(1+u^{\prime}\right)^{2}+\left(w^{\prime}\right)^{2}}
\end{aligned}
$$

According to Engesser's approach, the forces acting on the cross-section, $\breve{N}_{x}, \breve{Q}_{z}, \breve{M}_{y}$, are defined as shown in Fig. 2.

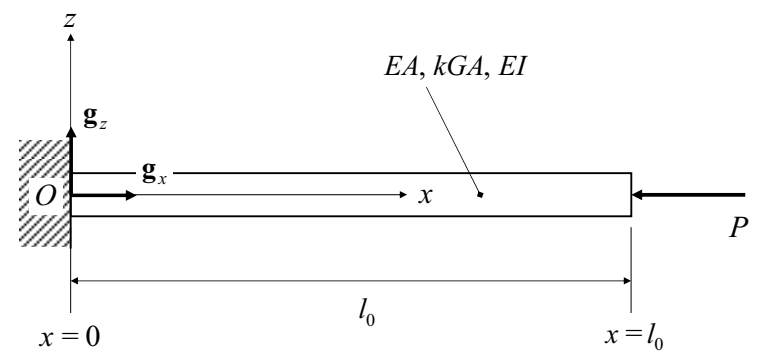

Fig. 1. Cantilever beam subjected to axial compression.

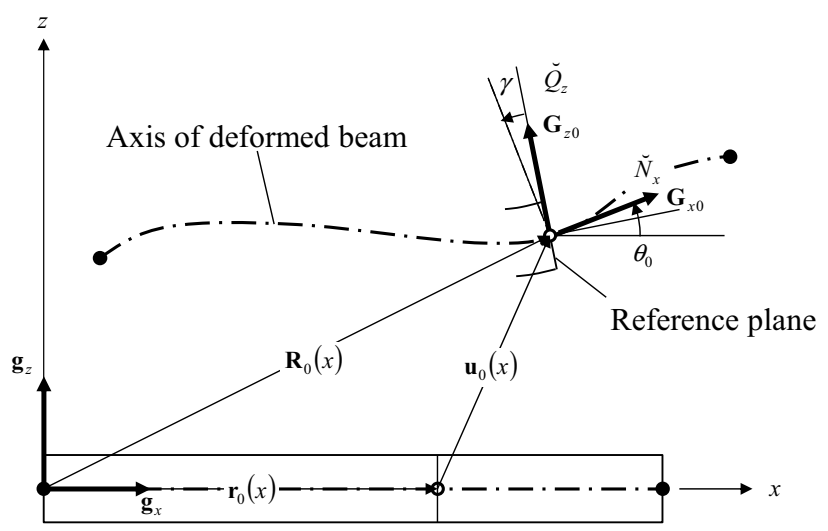

Fig. 2. Deformation of the beam axis.

The resultant force acting on a deformed cross-section is decomposed to $\breve{N}_{x}$, which is in the direction parallel to the axis, and $\breve{Q}_{z}$, which is in the direction parallel to the deformed cross-section. $\breve{M}_{y}$ is the bending moment acting on the deformed cross-section.

Engesser's generalized strains corresponding to the forces on the cross-section are defined as ${ }^{1)}$ :

$$
\begin{aligned}
& \breve{\varepsilon}_{x 0}=\sqrt{G_{x x 0}}-1=\sqrt{\left(1+u^{\prime}\right)^{2}+\left(w^{\prime}\right)^{2}}-1 \\
& \breve{\kappa}_{y}=-\left(\theta_{0}-\gamma\right)^{\prime} \\
& \breve{\gamma}_{x z 0}=\sqrt{G_{x x 0}} \sin \gamma=\sqrt{\left(1+u^{\prime}\right)^{2}+\left(w^{\prime}\right)^{2}} \sin \gamma
\end{aligned}
$$

where $\gamma$ is the angle between the normal line of the plane of the cross-section and the beam axis, as shown in Fig. 2.

\subsection{Constitutive equation}

The constitutive equation considered in this paper is the macroscopic relationship between forces on the cross-section (i.e., axial and shear forces and bending moment) and strains (i.e., axial strain, shear strain and curvature of beam axis).

$$
\breve{N}_{x}=E A \breve{\varepsilon}_{x 0}, \quad \breve{Q}_{z}=k G A \breve{\gamma}_{x z 0}, \quad \breve{M}_{y}=E I \breve{\kappa}_{y}
$$

where $E$ is Young's modulus, $G$ is shear modulus, $A$ is the cross-sectional area before deformation, $I$ is the moment of inertia of the cross-section before deformation, and $k$ is the shear correction factor.

\subsection{Definition of element coordinate system}

The beam is divided into finite elements. Each element has two grid points. The global coordinate system and the element coordinate systems are defined as shown in Fig. 4. The element span axis before deformation, $x_{e}$, starts from grid point 1 and extends through grid point 2 . The element 


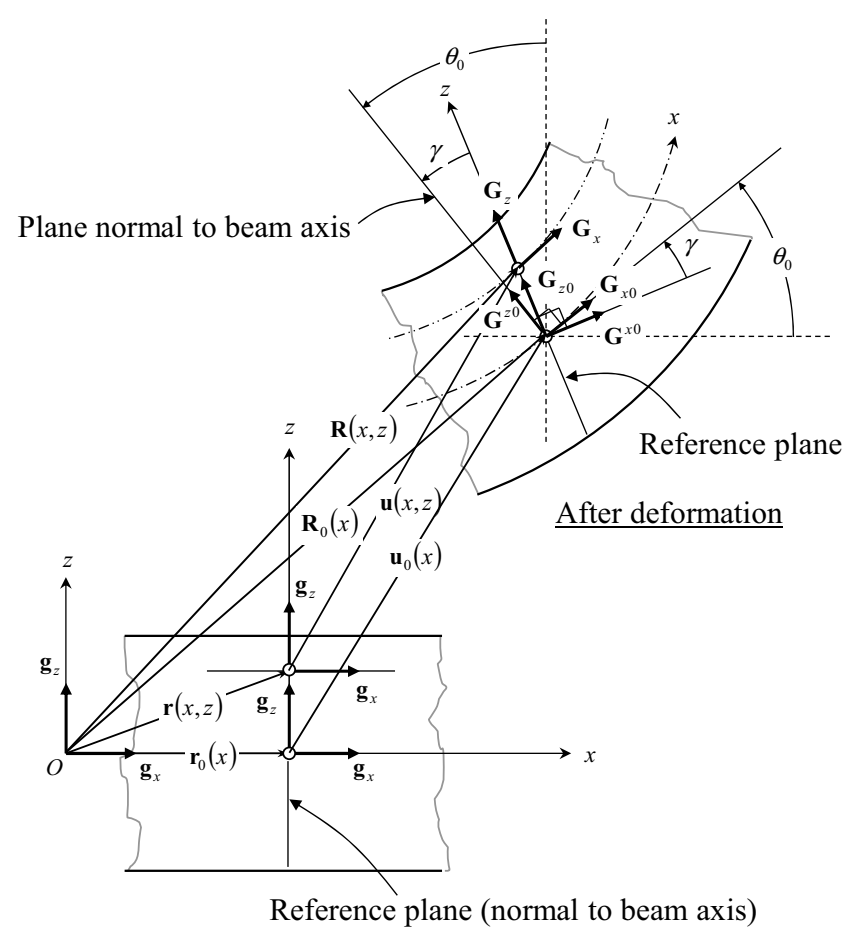

$\underline{\text { Before deformation }}$

Fig. 3. Deformed geometry of the extensible shearable elastica.

span axis after deformation, $\xi_{e}$, is defined in a similar manner. The angle between the $x_{e}$-axis and $\xi_{e}$-axis is $\phi_{e}$.

Grid point 1 of the undeformed element is moved onto grid point 1 of the deformed element, and the direction of the span axes are adjusted as shown in Fig. 5. The displacements of the deformed element in the element coordinate system are expressed with the displacements in the global coordinate system as follows.

$$
\begin{aligned}
& \phi_{e}=\tan ^{-1} \frac{w_{2}-w_{1}}{x_{2}+u_{2}-x_{1}-u_{1}} \\
& \theta_{0 e}=\theta_{0}-\phi_{e} \\
& \gamma_{e}=\gamma \\
& \Lambda_{e}=\sqrt{\left(x_{2}+u_{2}-x_{1}-u_{1}\right)^{2}+\left(w_{2}-w_{1}\right)^{2}} \\
& \xi_{e}=\left\{x+u(x)-x_{1}-u_{1}\right\} \cos \phi_{e}+\left\{w(x)-w_{1}\right\} \sin \phi_{e} \\
& \varsigma_{e}=-\left\{x+u(x)-x_{1}-u_{1}\right\} \sin \phi_{e}+\left\{w(x)-w_{1}\right\} \cos \phi_{e} \\
& u_{e}=\xi_{e}-x_{e}, \quad w_{e}=\varsigma_{e}
\end{aligned}
$$

Note that $u_{e 1}=w_{e 1}=w_{e 2}=0$. From Eq. (5), we obtain the following equation.

$$
u_{e 2}=\Lambda_{e}-L_{e}
$$

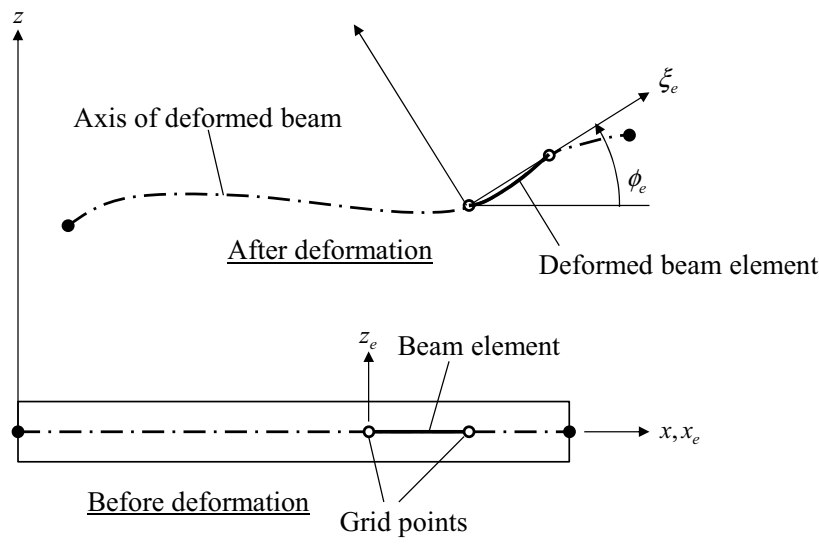

Fig. 4. Definition of the element coordinate systems.

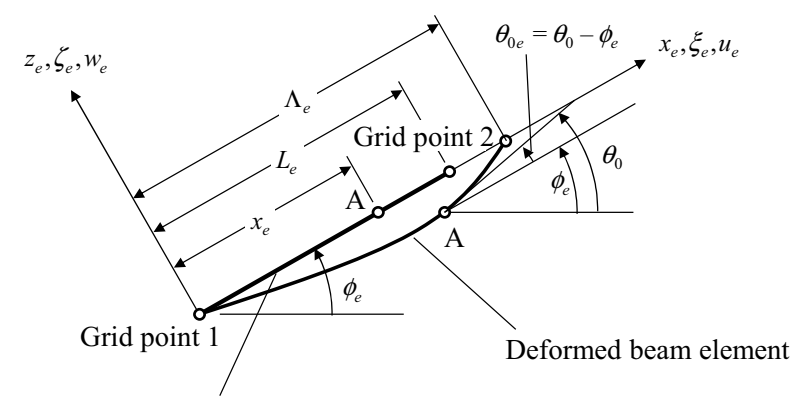

Undeformed beam element

Fig. 5. The relationship between undeformed and deformed elements.

If the beam is divided into enough elements, $\theta_{0 e}$ and $w_{e}$ become small. Then, the following equations are derived from Eq. (2) using the conditions of $\theta_{0 e} \ll 1, w_{e}^{\prime} \ll 1$ and $u_{e}^{\prime \prime} \ll 1$.

$$
\begin{aligned}
& \theta_{0 e} \cong \sin \theta_{0 e}=\frac{w_{e}^{\prime}}{\sqrt{\left(1+u_{e}^{\prime}\right)^{2}+\left(w_{e}^{\prime}\right)^{2}}} \cong \frac{w_{e}{ }^{\prime}}{1+u_{e}^{\prime}} \\
& \theta_{0 e}{ }^{\prime}=\frac{\left(1+u_{e}{ }^{\prime}\right) w_{e}^{\prime \prime}-u_{e}{ }^{\prime \prime} w_{e}{ }^{\prime}}{\left(1+u_{e}\right)^{2}+\left(w_{e}\right)^{2}} \cong \frac{w_{e}^{\prime \prime}}{1+u_{e}^{\prime}}
\end{aligned}
$$

\subsection{Interpolation of displacements in a beam element}

The displacement, $w_{e}$, in the deformed element coordinate system is interpolated by Hermite polynomials using the displacements and slopes of grid points, $w_{e 1}, w_{e 2}$ and $w_{e 1}{ }^{\prime}, w_{e 2}{ }^{\prime}$. The displacement, $u_{e}$, and the shear deformation angle, $\gamma_{e}$, in the deformed element coordinate system are interpolated by a linear function using the values at the grid points. The expression is as follows.

$$
\left[\begin{array}{c}
u_{e} \\
w_{e} \\
\gamma_{e}
\end{array}\right]=\left[\begin{array}{cccccc}
1-\frac{x_{e}}{L_{e}} & 0 & 0 & \frac{x_{e}}{L_{e}} & 0 & 0 \\
0 & x_{e}-2 \frac{x_{e}^{2}}{L_{e}}+\frac{x_{e}{ }^{3}}{L_{e}^{2}} & 0 & 0 & -\frac{x_{e}^{2}}{L_{e}}+\frac{x_{e}{ }^{3}}{L_{e}{ }^{2}} & 0 \\
0 & 0 & 1-\frac{x_{e}}{L_{e}} & 0 & 0 & \frac{x_{e}}{L_{e}}
\end{array}\right]\left[\begin{array}{c}
0 \\
w_{e 1}^{\prime} \\
\gamma_{e 1} \\
u_{e 2} \\
w_{e 2}^{\prime} \\
\gamma_{e 2}
\end{array}\right]
$$


Note that $u_{e 1}=w_{e 1}=w_{e 2}=0$ is used.

From Eq. (7), the following differentials are obtained.

$$
\begin{aligned}
& u_{e}^{\prime}=\frac{u_{e 2}}{L_{e}} \\
& w_{e}^{\prime}=\left(1-4 \frac{x_{e}}{L_{e}}+3 \frac{x_{e}{ }^{2}}{L_{e}^{2}}\right) w_{e 1}{ }^{\prime}+\left(-2 \frac{x_{e}}{L_{e}}+3 \frac{x_{e}{ }^{2}}{L_{e}{ }^{2}}\right) w_{e 2}{ }^{\prime} \\
& \gamma_{e}{ }^{\prime}=\frac{\gamma_{e 2}-\gamma_{e 1}}{L_{e}}
\end{aligned}
$$

Using Eqs. (6) and (8), we obtain the following expression.

$\theta_{0 e}=\left(1-4 \frac{x_{e}}{L_{e}}+3 \frac{x_{e}^{2}}{L_{e}^{2}}\right) \theta_{0 e 1}+\left(-2 \frac{x_{e}}{L_{e}}+3 \frac{x_{e}^{2}}{L_{e}^{2}}\right) \theta_{0 e 2}$

$\theta_{0 e^{\prime}}=\left(-\frac{4}{L_{e}}+6 \frac{x_{e}}{L_{e}^{2}}\right) \theta_{0 e 1}+\left(-\frac{2}{L_{e}}+6 \frac{x_{e}}{L_{e}^{2}}\right) \theta_{0 e 2}$

where $\theta_{0 e 1}$ and $\theta_{0 e 2}$ are the slopes of the axis of the element at grid points 1 and 2 in the element coordinate system (see Fig. 5). $\theta_{0 e}{ }^{\prime}$ and $\theta_{0 e}{ }^{\prime \prime}$ are used in lieu of $w_{e}{ }^{\prime}$ and $w_{e}{ }^{\prime \prime}$.

Table 1 shows the relationship between parameters for the undeformed and deformed elements. The displacement of the grid points in the global coordinates, $u_{i}, w_{i}, \gamma_{i}$, and $\theta_{0 i}$, are continuous at each grid point.

\subsection{Strain energy}

The strain energy is expressed as follows.

$U=\int_{0}^{l_{0}}\left[\frac{1}{2} E A \breve{\varepsilon}_{x 0 e^{2}}+\frac{1}{2} E I \breve{\kappa}_{y e}^{2}+\frac{1}{2} k G A \breve{\gamma}_{x z 0 e}{ }^{2}\right] d x$

Since the displacement, $w_{e}$, is much smaller than $u_{e}$ in the element coordinate system, $w_{e}^{\prime}$ and $w_{e}{ }^{\prime \prime}$ can be neglected. From Eqs. (3), (8) and (9), the strains in Eq. (10) are written as follows.

$$
\begin{aligned}
\breve{\varepsilon}_{x 0 e}= & \sqrt{\left(1+u_{e}{ }^{\prime}\right)^{2}+\left(w_{e}{ }^{\prime}\right)^{2}}-1 \cong u_{e}{ }^{\prime}=\frac{u_{e 2}}{L_{e}} \\
\breve{\kappa}_{y e}= & -\left(\theta_{0 e}-\gamma_{e}\right)^{\prime} \\
= & -\theta_{0 e^{\prime}}+\gamma_{e}^{\prime} \\
= & -\left(-\frac{4}{L_{e}}+6 \frac{x_{e}}{L_{e}{ }^{2}}\right) \theta_{0 e 1}-\left(-\frac{2}{L_{e}}+6 \frac{x_{e}}{L_{e}{ }^{2}}\right) \theta_{0 e 2} \\
& +\frac{\gamma_{e 2}-\gamma_{e 1}}{L_{e}} \\
\breve{\gamma}_{x z 0 e}= & \sqrt{\left(1+u_{e}\right)^{2}+\left(w_{e}\right)^{2}} \sin \gamma_{e} \\
\cong & \left(1+u_{e}^{\prime}\right) \sin \gamma_{e} \\
= & \left(1+\frac{u_{e 2}}{L_{e}}\right) \sin \left[\left(1-\frac{x_{e}}{L_{e}}\right) \gamma_{e 1}+\left(\frac{x_{e}}{L_{e}}\right) \gamma_{e 2}\right]
\end{aligned}
$$

The strain energy of the element, $U_{e}$, can be expressed with the strains as follows.
Table 1. The relationship between undeformed and deformed elements.

\begin{tabular}{llccc}
\hline & & & Grid 1 & Grid 2 \\
\hline Before & Global coordinate & $x$ & $x_{1}$ & $x_{2}$ \\
deformation & system & $z$ & 0 & 0 \\
\cline { 2 - 5 } & Element coordinate & $x_{e}$ & 0 & $L_{e}$ \\
& system & $z_{e}$ & 0 & 0 \\
\hline After & Global coordinate & $x$ & $x_{1}+u_{1}$ & $x_{2}+u_{2}$ \\
deformation & system & $z$ & $w_{1}$ & $w_{2}$ \\
& & $\gamma$ & $\gamma_{1}$ & $\gamma_{2}$ \\
& & $\theta_{0}$ & $\theta_{01}$ & $\theta_{02}$ \\
\cline { 2 - 5 } & Element coordinate & $\xi_{e}$ & 0 & $\Lambda_{e}$ \\
& system & $\zeta_{e}$ & 0 & 0 \\
& & $\gamma_{e}$ & $\gamma_{e 1}=\gamma_{1}$ & $\gamma_{e 2}=\gamma_{2}$ \\
& $\theta_{0 e}$ & $\theta_{0 e 1}=\theta_{01}-\phi_{e}$ & $\theta_{0 e 2}=\theta_{02}-\phi_{e}$ \\
\hline
\end{tabular}

$$
\begin{aligned}
U_{e}= & \frac{1}{2} E_{e} A_{e} \int_{0}^{L_{e}}\left(\breve{\varepsilon}_{x 0 e}\right)^{2} d x_{e}+\frac{1}{2} E_{e} I_{e} \int_{0}^{L_{e}}\left\{\left(\theta_{0 e}-\gamma_{e}\right)^{\prime}\right\}^{2} d x_{e} \\
& +\frac{1}{2} k G_{e} A_{e} \int_{0}^{L_{e}}\left(\breve{\gamma}_{x z 0}\right)^{2} d x_{e}
\end{aligned}
$$

Substituting Eq. (11) into Eq. (12), the strain energy of the element is expressed with the grid point displacements as follows.

$$
\begin{aligned}
U_{e}= & \frac{E_{e} A_{e} L_{e}}{2}\left(\frac{u_{e 2}}{L_{e}}\right)^{2} \\
& +\frac{k G_{e} A_{e} L_{e}}{4}\left(1+\frac{u_{e 2}}{L_{e}}\right)^{2}\left\{1-\frac{\sin 2 \gamma_{e 2}-\sin 2 \gamma_{e 1}}{2\left(\gamma_{e 2}-\gamma_{e 1}\right)}\right\} \\
& +\frac{E_{e} I_{e}}{2 L_{e}}\left\{\begin{array}{l}
4\left(\theta_{0 e 1}{ }^{2}+\theta_{0 e 2}{ }^{2}+\theta_{0 e 1} \theta_{0 e 2}\right) \\
-2\left(\theta_{0 e 2}-\theta_{0 e 1}\right)\left(\gamma_{e 2}-\gamma_{e 1}\right)+\left(\gamma_{e 2}-\gamma_{e 1}\right)^{2}
\end{array}\right\}
\end{aligned}
$$

Note that $w_{e}{ }^{\prime}$ and $w_{e}{ }^{\prime \prime}$ are not necessary to calculate the strain energy of the element, because $w_{e}{ }^{\prime}$ and $w_{e}{ }^{\prime \prime}$ do not appear in Eq. (13) or Table 1.

\subsection{Principle of stationary potential energy}

The potential energy of the beam, $\Pi$, is the sum of the strain energy of all the elements minus the work done by the external forces.

$$
\Pi=U-P u\left(l_{0}\right)=\sum_{\text {all elements }} U_{e}-P u\left(l_{0}\right)
$$

where $P$ is the external force acting on the free end of the cantilever beam (positive for positive $x$-direction).

The optimization tool, "Solver" in MS-Excel, is used to solve the problem. A set of the grid point displacements in the global coordinate system, $u_{i}, w_{i}, \theta_{0 i}, \gamma_{i}: i=1, \ldots n$ ( $n$ is total number of grid points), are assumed as initial values. The displacements are transformed to the element coordinates and the potential energy is calculated using MS-Excel. The potential energy is assigned as the "objective cell" to minimize and the grid point displacements are assigned as "variable cells." Run "Solver" and a set of displacements to minimize the potential energy is obtained. Because the problem is nonlinear, the solutions are dependent on the initial values. It is necessary to choose a proper set of initial values.

The details of the solution process are shown in Fig. 6. A 


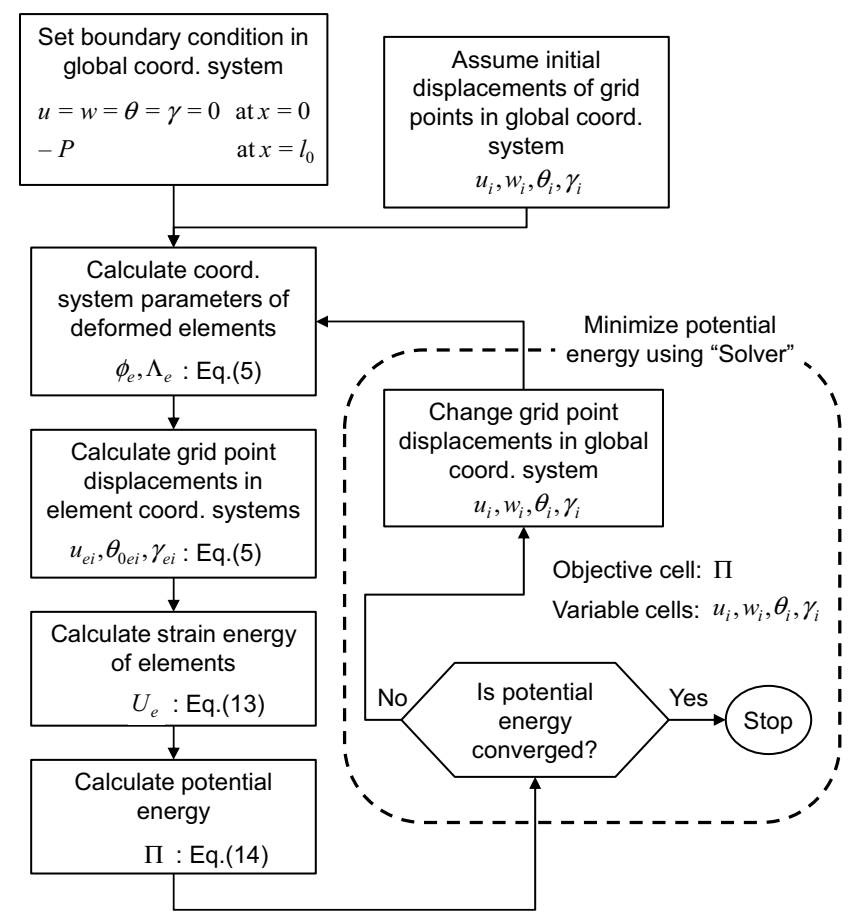

Fig. 6. Solution process in MS-Excel.

matrix calculation is not used in the process. Since "Solver" conducts minimization of the potential energy (i.e., righthand part of Fig. 6), programming is not necessary. The pre- and post-process of the results are easy because the analysis is performed using the MS-Excel spreadsheet. These features are the advantage of this method over non-linear finite element analysis.

\subsection{Grid point forces}

The grid point forces of the element shown in Fig. 7 are expressed as follows.

From Eqs. (4) and (11), the axial forces are,

$$
\begin{aligned}
& \breve{N}_{x e 1}=-E A \breve{\varepsilon}_{x 0 e}=-\frac{E A}{L_{e}} u_{e 2} \\
& \breve{N}_{x e 2}=E A \breve{\varepsilon}_{x 0 e}=\frac{E A}{L_{e}} u_{e 2}
\end{aligned}
$$

The shear forces are,

$$
\begin{aligned}
& \breve{Q}_{z e 1}=-k G A \breve{\gamma}_{x z 0 e 1}=-k G A\left(1+\frac{u_{e 2}}{L_{e}}\right) \sin \gamma_{e 1} \\
& \breve{Q}_{z e 2}=k G A \breve{\gamma}_{x z 0 e 2}=k G A\left(1+\frac{u_{e 2}}{L_{e}}\right) \sin \gamma_{e 2}
\end{aligned}
$$

The bending moments are expressed as follows.

$\breve{M}_{y e 1}=-E I \breve{\kappa}_{y e 1}=\frac{E I}{L_{e}}\left(-4 \theta_{0 e 1}-2 \theta_{0 e 2}-\gamma_{e 2}+\gamma_{e 1}\right)$

$\breve{M}_{y e 2}=E I \breve{\kappa}_{y e 2}=-\frac{E I}{L_{e}}\left(2 \theta_{0 e 1}+4 \theta_{0 e 2}-\gamma_{e 2}+\gamma_{e 1}\right)$

These forces are transformed to the global coordinate system as follows.

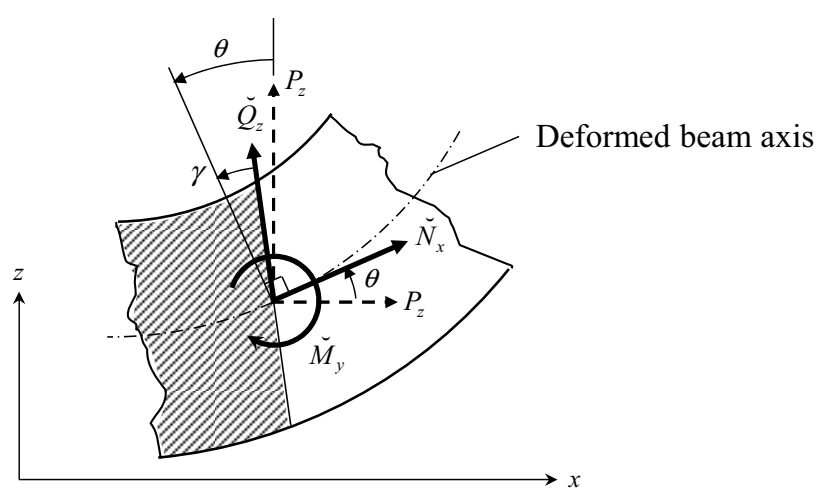

Fig. 7. Direction of forces in Engesser's approach.

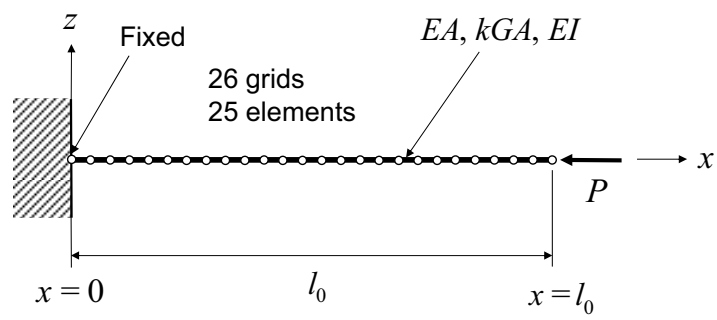

Fig. 8. Cantilever beam subjected to end load.

$$
\begin{aligned}
& P_{x e i}=\breve{N}_{x e i} \cos \theta_{0 e i}-\breve{Q}_{z e i} \sin \left(\theta_{0 e i}-\gamma_{e i}\right) \\
& P_{z e i}=\breve{N}_{x e i} \sin \theta_{0 e i}+\breve{Q}_{z e i} \cos \left(\theta_{0 e i}-\gamma_{e i}\right) \\
& \quad: i=1,2
\end{aligned}
$$

\section{Numerical Analysis}

Numerical analysis of the straight cantilever beam with a constant cross-section, shown in Fig. 8, is conducted.

\subsection{Model}

The cantilever beam is divided into 25 elements and 26 grid points, as shown in Fig. 8.

\subsection{Buckling loads}

Buckling loads for various combinations of axial stiffness and shear stiffness are obtained using the energy method. The buckling loads are determined from the load-lateral displacement relationship, as shown in Fig. 9. Normalized applied load is used as the ordinate and the square of normalized lateral displacement is used as the abscissa. The plotted points are extrapolated toward zero and the buckling load is obtained as an intercept point at the ordinate axis.

A comparison between the buckling loads obtained using the energy method and the equation in Taki and Kondo ${ }^{1)}$ (see Eq. (19)) is shown in Figs. 10 and 11. The open circles in the figures are the buckling loads obtained using the energy method. The crosses show the minimum loads obtained using the energy method, and they are smaller than the buckling loads. See the next section for an explanation of the minimum loads. The agreement between the buckling load using the energy method and the equation is very good. 


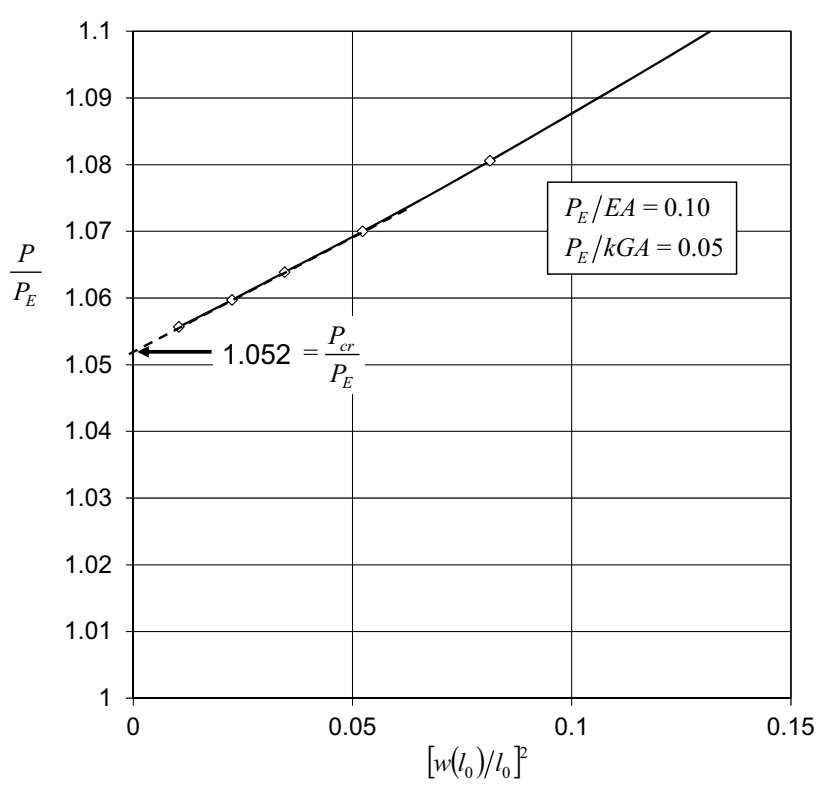

Fig. 9. Determination of buckling load.

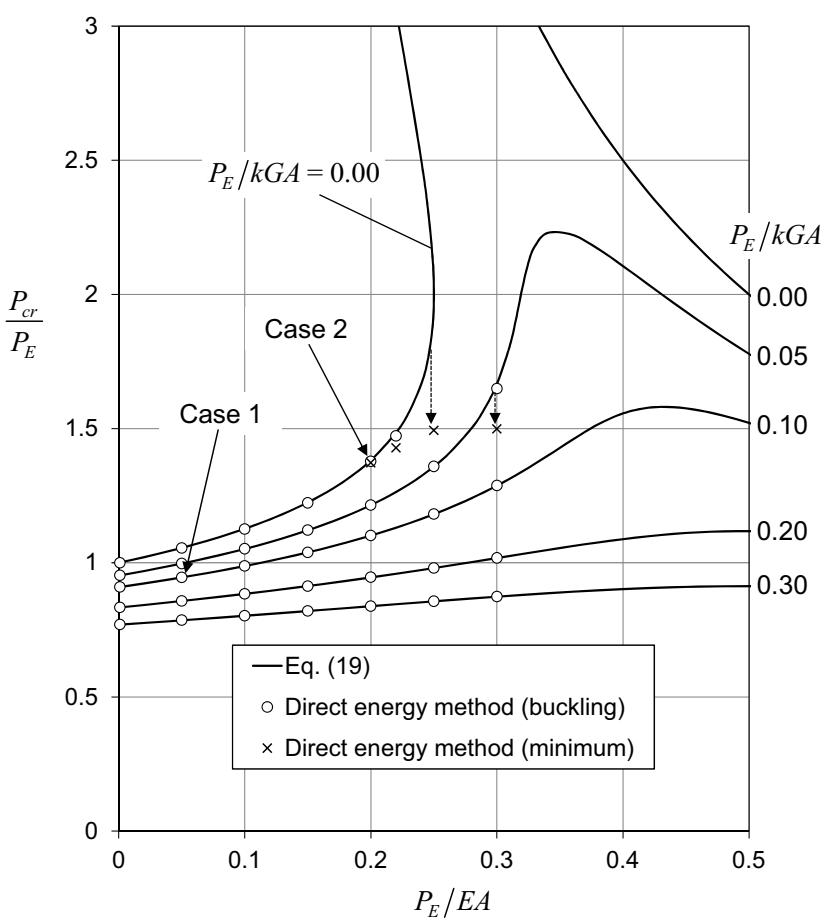

Fig. 10. Comparison of buckling loads—effect of axial stiffness.

$$
\begin{aligned}
& \left(\frac{P_{E}}{E A}\right)^{2}\left(\frac{P_{E n(n)}}{P_{E}}\right)^{3}-2\left(\frac{P_{E}}{E A}\right)\left(\frac{P_{E n(n)}}{P_{E}}\right)^{2} \\
& +\left\{1+(2 n-1)^{2}\left(\frac{P_{E}}{E A}+\frac{P_{E}}{k G A}\right)\right\}\left(\frac{P_{E n(n)}}{P_{E}}\right) \\
& -(2 n-1)^{2}=0
\end{aligned}
$$

where $P_{E n(n)}$ is the buckling load of the $n$-th mode of Engesser's approach and $P_{E}=\pi^{2} E I /\left(4 l_{0}^{2}\right)$ is the Euler buckling load of the first mode.

\subsection{Load-displacement relationship}

The load-displacement curves for representative combina-

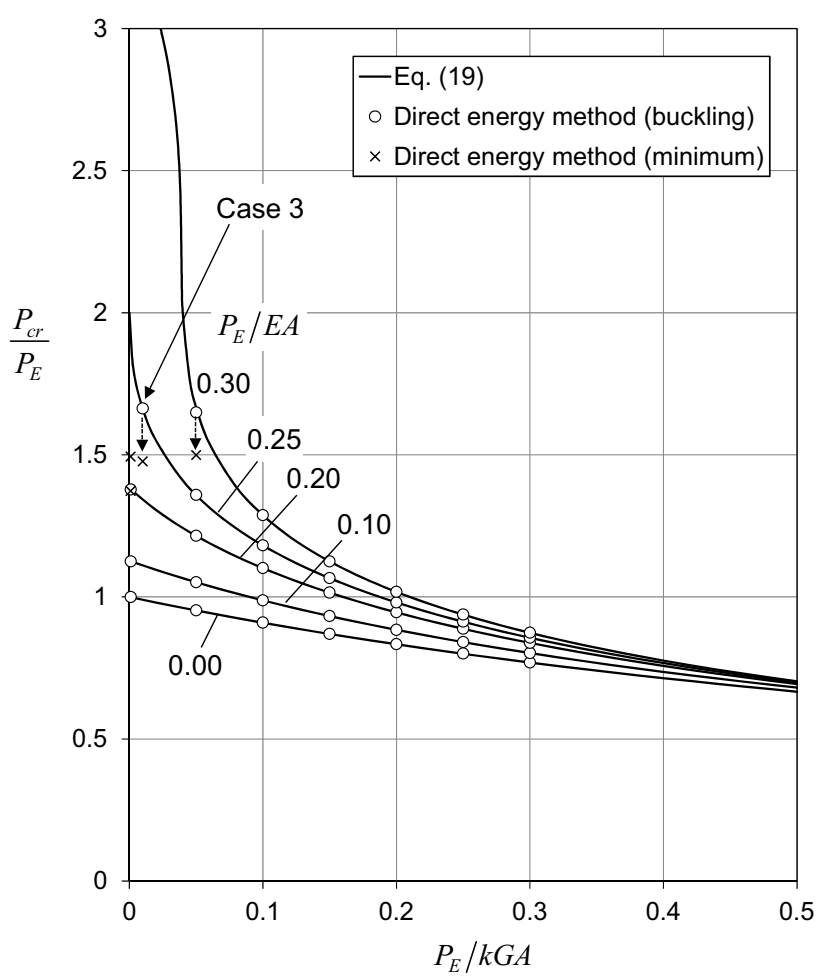

Fig. 11. Comparison of buckling loads - effect of shear stiffness.
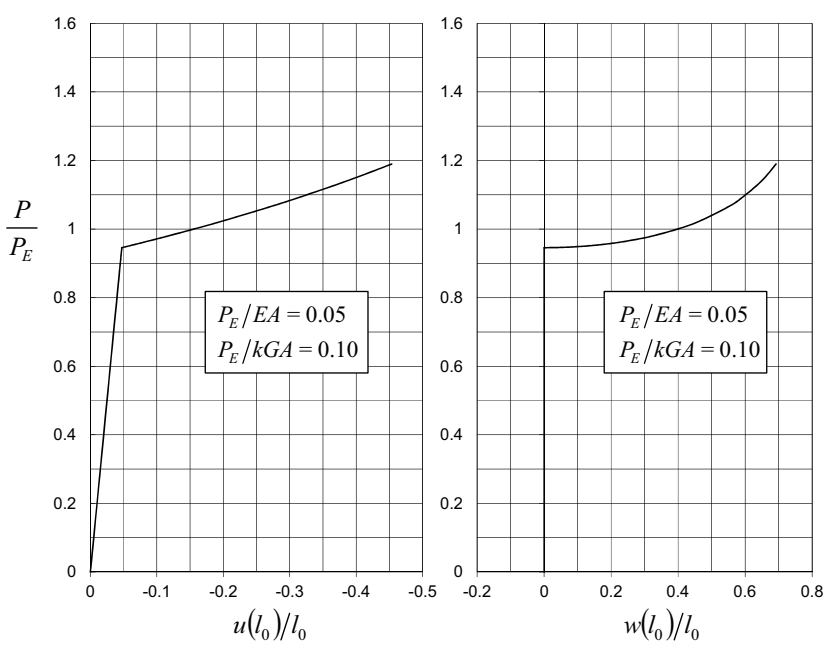

Fig. 12. Load-displacement relationship_Case 1.

tions of axial stiffness and shear stiffness are calculated using the energy method. Figures 12 to 14 show the results for beams with the following stiffness.

Case 1: $P_{E} / E A=0.05, P_{E} / k G A=0.10$

Representative beam with low shear stiffness (see Fig. 10)

Case 2: $P_{E} / E A=0.20, P_{E} / k G A=0.001$

Representative beam with indistinct snap-through behavior (see Fig. 10)

Case 3: $P_{E} / E A=0.25, P_{E} / k G A=0.01$

Representative beam with distinct snap-through behavior (see Fig. 11)

Note that the minimum loads smaller than the buckling loads exist in the load-displacement curves in Figs. 13 and 14. Snap-through behavior occurs in these load-displacement 
Trans. Japan Soc. Aero. Space Sci., Vol. 60, No. 5, 2017
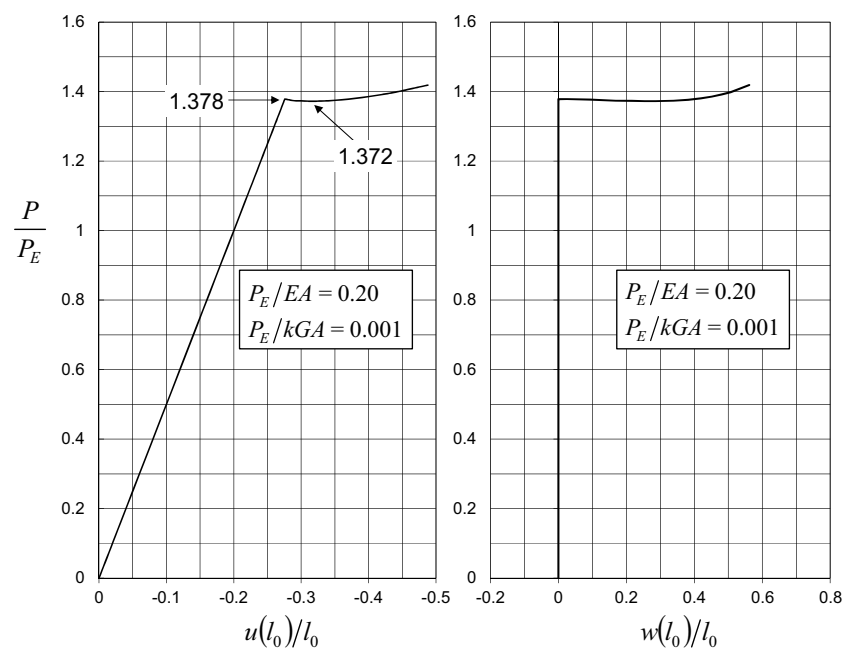

Fig. 13(a). Load-displacement relationship—Case 2.
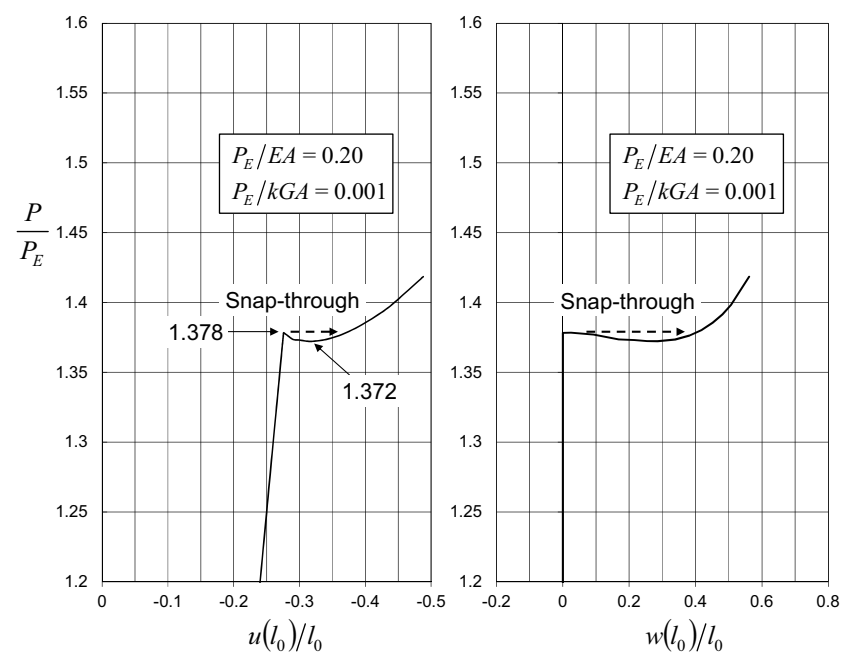

Fig. 13(b). Load-displacement relationship-Case 2 (enlarged).

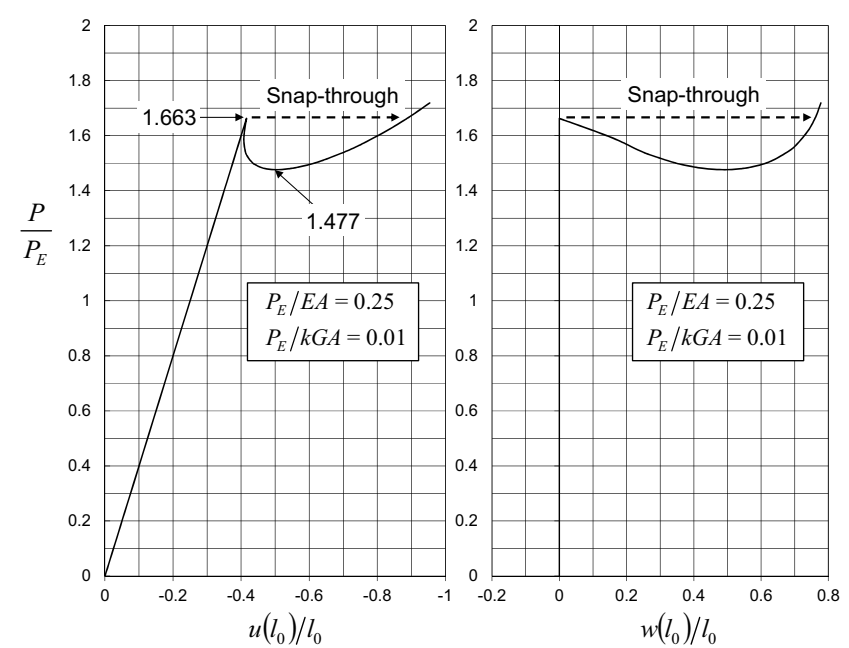

Fig. 14. Load-displacement relationship-Case 3.

curves. The existence of the snap-through behavior was reported by Magnusson et al. ${ }^{2)}$ for extensible elastica. According to Magnusson et al., the smallest value of $P_{E} / E A$ for extensible elastica that has snap-through is $P_{E} / E A=3 / 16=$ 0.1875 . The smallest value of $P_{E} / E A$ increases as the value

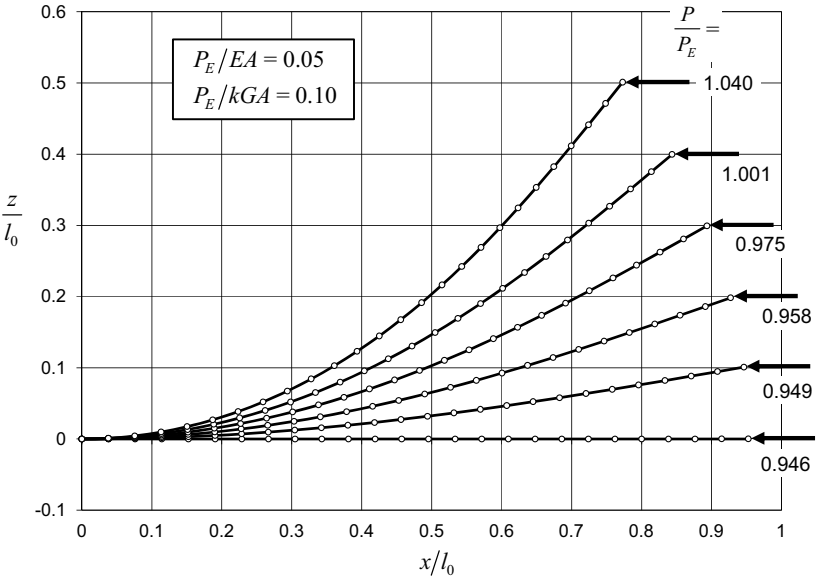

Fig. 15. Post-buckling deformation-Case 1 .

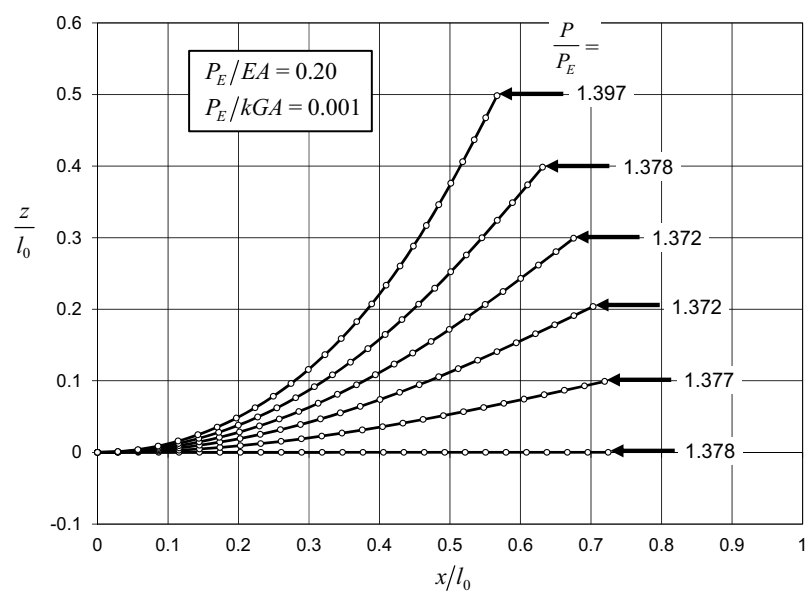

Fig. 16. Post-buckling deformation-Case 2 .

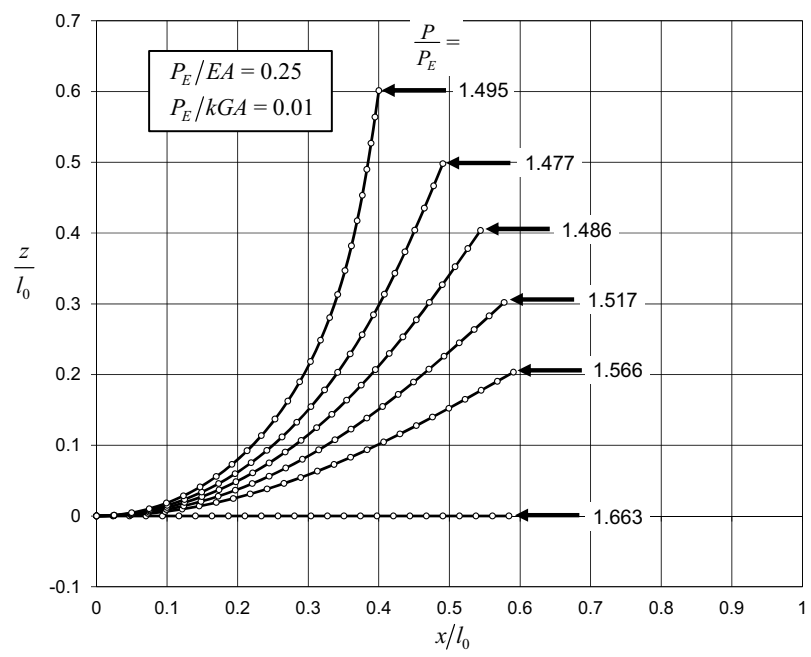

Fig. 17. Post-buckling deformation-Case 3 .

of $P_{E} / k G A$ increases. Snap-through does not occur at $P_{E} / E A=0.20$ with $P_{E} / k G A=0.05$ and 0.10 , as shown in Fig. 10. Note that the condition of $P_{E} / E A \approx 0.20$ is extremely elastic and this kind of condition exists only in analysis.

Deformed forms of the beams are shown in Figs. 15 to 17. The values of the end forces are also shown in the figures. 


\section{Conclusions}

A direct energy minimization method is proposed to solve the post-buckling behavior of extensible and shearable beams subjected to axial compression load. The features of the method are as follows.

- Programing is not required.

- Data input and post-processing are easy because the analysis is performed using an MS-Excel spreadsheet.

The buckling loads calculated by the method are in good agreement with the analytical solution reported in Taki and Kondo. ${ }^{1)}$ Load-displacement curves for extensible shearable elastica are obtained. Snap-through behavior is observed in the load-displacement curves of beams with low axial stiffness.

\section{References}

1) Taki, T. and Kondo, K.: Variational Principle of Extensible Shearable Elastica-Engesser's Approach, Trans. Jpn. Soc. Aeronaut. Space Sci., 60 (2017), pp. 284-294.

2) Magnusson, A., Ristinmaa, M., and Ljung, C.: Behaviour of the Extensible Elastica Solution, Int. J. Solid Struct., 38 (2001), pp. 8441-8457.

3) Kuramoto, K. and Kondo, K.: Finite Element Analysis for Extensible Elastica, Presented at the 48th JSASS/JSME/JAXA Structures Conference, 2006, Kobe, Japan, pp. 135-137 (in Japanese).

4) Humer, A.: Exact Solutions for the Buckling and Postbuckling of ShearDeformable Beams, Acta Mech., 224 (2013), pp. 1493-1525.

5) Taki, T.: Structural Analysis, Taki's Homepage at http:// www.geocities.jp/toshimi_taki/index.htm (accessed 7 August 2017).

Y. Miyazaki Associate Editor 